\title{
Seguimiento clínico al año de angioplastias coronarias exitosas en pacientes no seleccionados
}

\author{
Humberto Dighero $\mathrm{T}^{1}$, Jacobo Wajner $\mathrm{A}^{\mathbf{1}}$, Angel Puentes $\mathrm{R}^{\mathbf{1}}$, \\ Flavio Zepeda $\mathrm{M}^{1}$, Augusto Bellet $\mathrm{P}^{1}$, Hernán Donoso $\mathrm{P}^{1}$, \\ Arturo Guillem V ${ }^{1}$, Waldo Aranda $\mathrm{C}^{2 \mathrm{a}}$. \\ One year follow up of successful \\ coronary angioplasty \\ in non selected patients
}

Background: Re-stenosis after percutaneous Coronary Angioplasty (PTCA) is related to clinical and angiographic features. Aim: To describe the clinical and angiographic characteristic of our patients with coronary cardiopathy subjected to PTCA and the predictor factors for re-stenosis. Material and methods: We gathered the clinical and angiographic characteristics of all patients who underwent a successful PTCA of a native coronary artery. All patients had a clinical assessment one year after the procedure. Patients were classified in Group 1, if they did not have angina or coronary events after the angioplasty or Group 2, if they had angina or a coronary event after the procedure. Only Group 2 patients were subjected to a coronary angiogram. Results: We collected 383 PTCA procedures. Follow up information was obtained in $92.2 \%$. Three hundred forty two patients $(89.3 \%)$ were assessed one year the procedure. Nine patients (2.3\%) died of a cardiovascular cause. Ninety patients (26.3\%) were classified in Group 2. In 65 patients, angiographic re-stenosis was demonstrated (19\%). Restenosis occurred in 36 and 13\% of patients with an without Diabetes Mellitus, respectively ( $p$ $<0.01)$. The other clinical predictor variables were a history of myocardial infarction $(p=0.007)$, obesity ( $p=0.041$ ) and hypercholesterolemia $(p=0.050)$. None of the angiographic characteristics predicted restenosis. Stents were protective factors against restenosis (15.6\% in stented lesions vs $25.4 \%$ in nonstented; $p=0.01$ ). Conclusions: Re-stenosis after angioplasty occured in $19 \%$ of our patients with angina or coronary events. The clinical variables associated with a higher risk of restenosis were diabetes (the main risk factor), previous myocardial infarction, obesity and hypercholesterolemia. Angiographic variables were not associated with re-stenosis. The use of stents decreases the incidence of re-stenosis in all groups (Rev Méd Chile 2006; 134: 1383-92).

(Key words: Angioplasty, balloon; Coronary angiography; Coronary arteriosclerosis)

Recibido el 13 de octubre, 2005. Aceptado el 25 abril, 2006.

${ }^{1}$ Departamento de Cardiología, Servicio de Medicina Interna, Hospital San Juan de Dios, Servicio de Salud Metropolitano Occidente. ${ }^{2}$ Escuela de Salud Pública, Universidad de Chile.

a Profesor de Matemática y Estadística.

Correspondencia a: Dr. Humberto Dighero T. Hospital San Juan de Dios. Huérfanos 3255, Santiago Centro. Santiago de Chile. Teléfono: 5742017. Fax: 6818039. E mail: hdighero@hsjd.cl. 
L a angioplastia coronaria transluminal percutánea (ACTP), introducida en 1977, cambió el modo de enfrentar la cardiopatía coronaria, contribuyendo en la disminución de la mortalidad y morbilidad secundarias derivadas de la misma. Sin embaryo, sus complicaciones mediatas ensombrecieron el optimismo inicial y motivaron la búsqueda de soluciones, de las cuales, el stent, introducido en 1986, se convirtió en su complemento ideal. No obstante, la reestenosis continúa presentándose en un porcentaje significativo de casos, a pesar de los innumerables avances tecnológicos en el diseño y composición de los stents, como de nuevas técnicas destinadas a combatirla.

Diversos factores, como la diabetes mellitus ${ }^{1-5}$, angioplastia de lesiones largas ${ }^{6}$, de pequeños vasos $<3 \mathrm{~mm}^{7,8}$, de oclusiones completas ${ }^{7}$, de lesiones en bifurcación ${ }^{9}$ y otros, han sido asociados a una mayor probabilidad de reestenosis.

El objetivo del presente trabajo es conocer las características de nuestra población portadora de cardiopatía coronaria que llega a ACTP, así como los factores clínicos y angiográficos que inciden en la reestenosis.

\section{Material y MÉTOdO}

El estudio se realizó en la Unidad de Hemodinamia y Departamento de Cardiología del Hospital San Juan de Dios, pertenecientes al Servicio de Salud Metropolitano Occidente de Santiago de Chile.

Del total de ACTP realizadas entre enero de 1996 y diciembre de 2000, se seleccionaron las exitosas, correspondientes a lesiones de arterias nativas. La población estudiada quedó representada por 383 pacientes en los que se rescataron las características clínicas (edad, sexo, presencia o no de diabetes mellitus, hipertensión arterial, dislipidemia, tabaquismo, obesidad y el antecedente de infarto del miocardio) y angiográficas (tipo de lesión, largo, localización en bifurcación y presencia de stent). Los datos se obtuvieron de la entrevista personal, de la ficha clínica, del informe de la coronariografía y la angioplastia. Fueron excluidos del análisis las ACTP frustras y los fallecidos durante la hospitalización en la que se realizó la ACTP.
Se realizó un control clínico al año del procedimiento, clasificándolos en Grupo 1: pacientes sin angina y libres de eventos coronarios; y Grupo 2: pacientes con angina o eventos coronarios definidos (angina inestable o infarto agudo del miocardio). No se realizaron exámenes no invasivos para la clasificación de los pacientes. A la mayoría de los clasificados como Grupo 2 se les realizó una coronariografía control para certificar la reestenosis angiográfica. No se reestudiaron los pacientes del Grupo 1. Los fallecidos durante el período de seguimiento fueron clasificados como de causa cardiovascular o no, de acuerdo al diagnóstico del certificado de defunción.

Definiciones. Se consideró angioplastia exitosa toda angioplastia con una estenosis residual inferior a 50\% y flujo TIMI 2-3 como resultado final del procedimiento. Para el diagnóstico de obesidad, se consideraron los diagnósticos de sobrepeso $u$ obesidad plasmados en la ficha clínica. Las demás patologías crónicas o factores de riesgo cardiovascular debían estar consignadas en ella. Infarto previo fue todo evento coronario que cumpliera con los criterios clásicos de infarto (clínico, electrocardiográfico, enzimático o todos) o la existencia del antecedente referido por el paciente más signos electrocardiográficos de necrosis, ocurrido hasta la fecha de la coronariografía diagnóstica. Las características angiográficas se clasificaron de la siguiente forma: 1) tipo de lesión, en lesiones A, B1, B2 y C de acuerdo a la Clasificación de la American Heart Association/American College of Cardiology y subclasificación de Ellis y cols ${ }^{10,11}$; 2) lesiones no complejas y lesiones complejas a la combinación de las lesiones $\mathrm{A}+\mathrm{B} 1$ y $\mathrm{B} 2+\mathrm{C}$, respectivamente y 3 ) largo de la lesión, en lesiones cortas si éstas eran iguales o menores a $15 \mathrm{~mm}$ y lesiones largas si eran mayores de $15 \mathrm{~mm}$. Se consideró reestenosis angiográfica la presencia de estenosis comnaria en $50 \%$ o más de la lesión tratada. Fallecido de causa cardiovascular, fue todo fallecido que contara como causa primaria de muerte los siguientes diagnósticos: muerte súbita, infarto agudo del miocardio, síndrome coronario agudo, insuficiencia cardíaca 0 edema agudo del pulmón.

Se analizan las características de la población y los factores clínicos y angiográficos que inciden en la reestenosis posangioplastia. 
Estadística. En el análisis estadístico se usó el programa estadístico Stata en un modelo de regresión logística múltiple, considerando como significativa una asociación entre una variable predictora y la variable reestenosis, cuando el Odds Ratio (O.R) tiene asociado un valor de $\mathrm{p}$ igual o menor a 0,05.

El estudio fue aprobado por el comité de ética del Hospital «San Juan de Dios».

\section{RESULTADOS}

En el período estudiado, se realizaron un total de 554 ACTP, 484 exitosas (87,36\%) y $70(12,64 \%)$ frustras. La distribución de las ACTP por años se representa en la Figura 1. Al seleccionar las ACTP de arterias nativas de pacientes que sobrevivieron a la hospitalización y obtener los datos requeridos, la muestra quedó constituida por 383 ACTP.

Las características clínicas de la población que llegó a ACTP (383 pacientes) se describen en la Tabla 1. El promedio de edad fue de 62 años (rango 30-80), 73,9\% eran hombres, 29\% eran diabéticos, 51\% hipertensos, 35,8\% dislipidémicos, $20,9 \%$ obesos, $53 \%$ eran fumadores y $60,8 \%$ tenía el antecedente de infarto del miocardio. En la Tabla 2 se resume las características angiográficas. El 61,4\%
Tabla 1. C aracterísticas clínicas de 383 pacientes

\begin{tabular}{|lrr|}
\hline Característica & Número & $\%$ \\
\hline Sexo & & \\
$\quad$ Masculino & 283 & 73,9 \\
Diabetes & 111 & 29 \\
Hipertensión arterial & 195 & 51 \\
Hipercolesterolemia & 137 & 35,8 \\
Obesidad & 80 & 20,9 \\
Tabaco & 203 & 53 \\
Antecedentes de infarto & 233 & 60,8 \\
\hline
\end{tabular}

Tabla 2. C aracterísticas angiográficas de 383 pacientes

\begin{tabular}{|lrc|}
\hline Característica & Número & $\%$ \\
\hline Tipo de lesión & & \\
A+B1 & 148 & 38,64 \\
B2+C & 235 & 61,36 \\
Lesiones cortas & 219 & 57,18 \\
Lesiones largas & 164 & 42,82 \\
Lesiones en bifurcación & 64 & 16,71 \\
Lesiones con stent & 251 & 65,53 \\
\hline
\end{tabular}

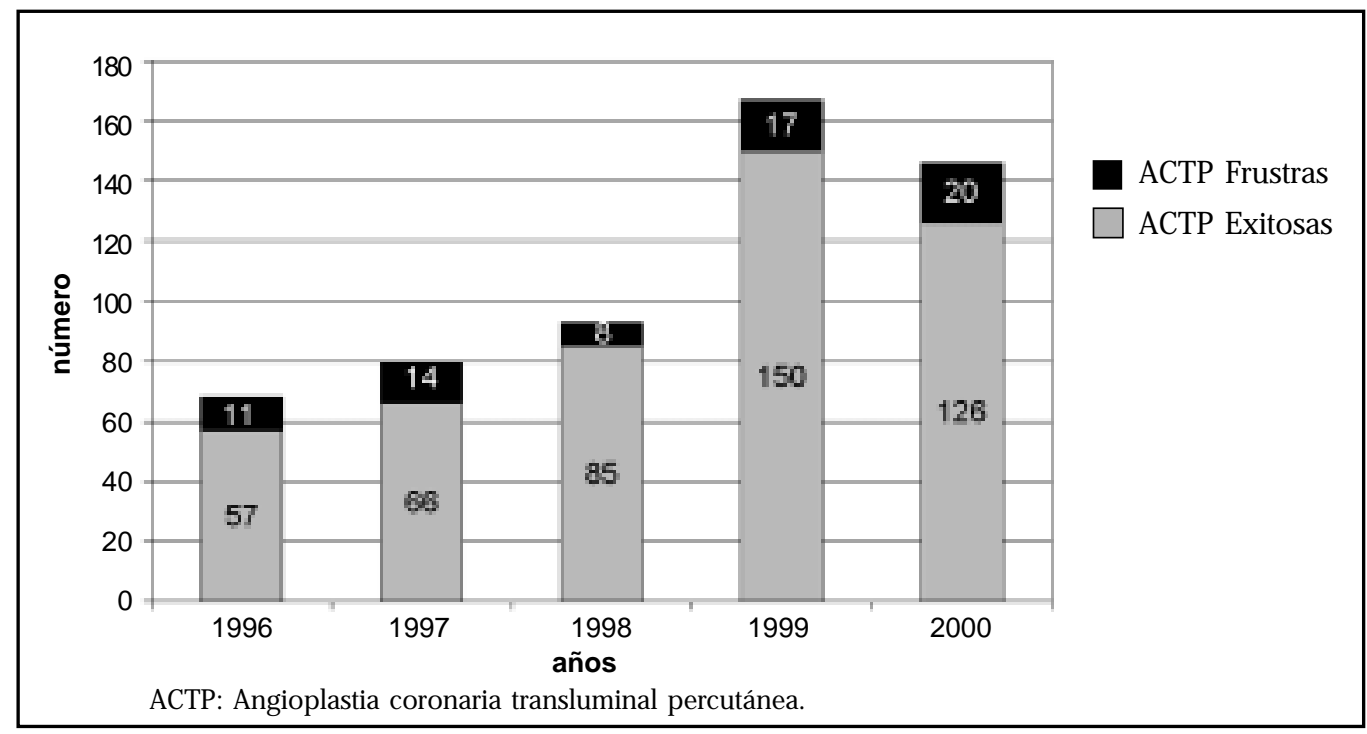

Figura 1. Distribución de angioplastias por años. 
de las lesiones eran complejas, 42,8\% eran largas, sólo $16,7 \%$ se encontraban en bifurcación y en $65,5 \%$ de las angioplastias se instaló stent.

En 353 pacientes $(92,2 \%)$ se obtuvo información del seguimiento. Once (2,9\%) fallecieron en este período, nueve (2,3\%) de causa cardiovascular. En $342(89,3 \%)$ se realizó un control clínico al año de la ACTP, clasificándolos en Grupo 1 o Grupo 2 y conformando la muestra final sobre la que se realizó el análisis de las variables clínicas y angiográficas relacionadas con la reestenosis. Treinta pacientes fueron perdidos de seguimiento $(7,8 \%)$.

Noventa pacientes (26,3\% de 342) presentaron angina 0 eventos coronarios durante el período de seguimiento (Grupo 2); de ellos, 85 (94,4\% de los pacientes del Grupo 2) fueron estudiados con nueva coronariografía. En 65 se demostró reestenosis angiográfica (19\% de 342) (Figura 2).

La diabetes mellitus fue la variable clínica más relacionada con la reestenosis angiográfica, presentándose en 33 pacientes de 91 diabéticos $(36,26 \%)$ y sólo en $12,75 \%$ de 251 no diabéticos (32 pacientes; $p$ $\varangle 0,0001)$. Las otras variables con significado estadístico fueron, por orden de importancia, el antecedente de infarto del miocardio ( $p=0,007$ ), la obesidad ( $p$ $=0,041)$ y marginalmente, la dislipidemia ( $p=0,050$ ). No influyeron en la reestenosis las variables sexo, hipertensión arterial y tabaquismo (Tabla 3).

No se demostró influencia de las características angiográficas (tipo de lesión, largo, localización

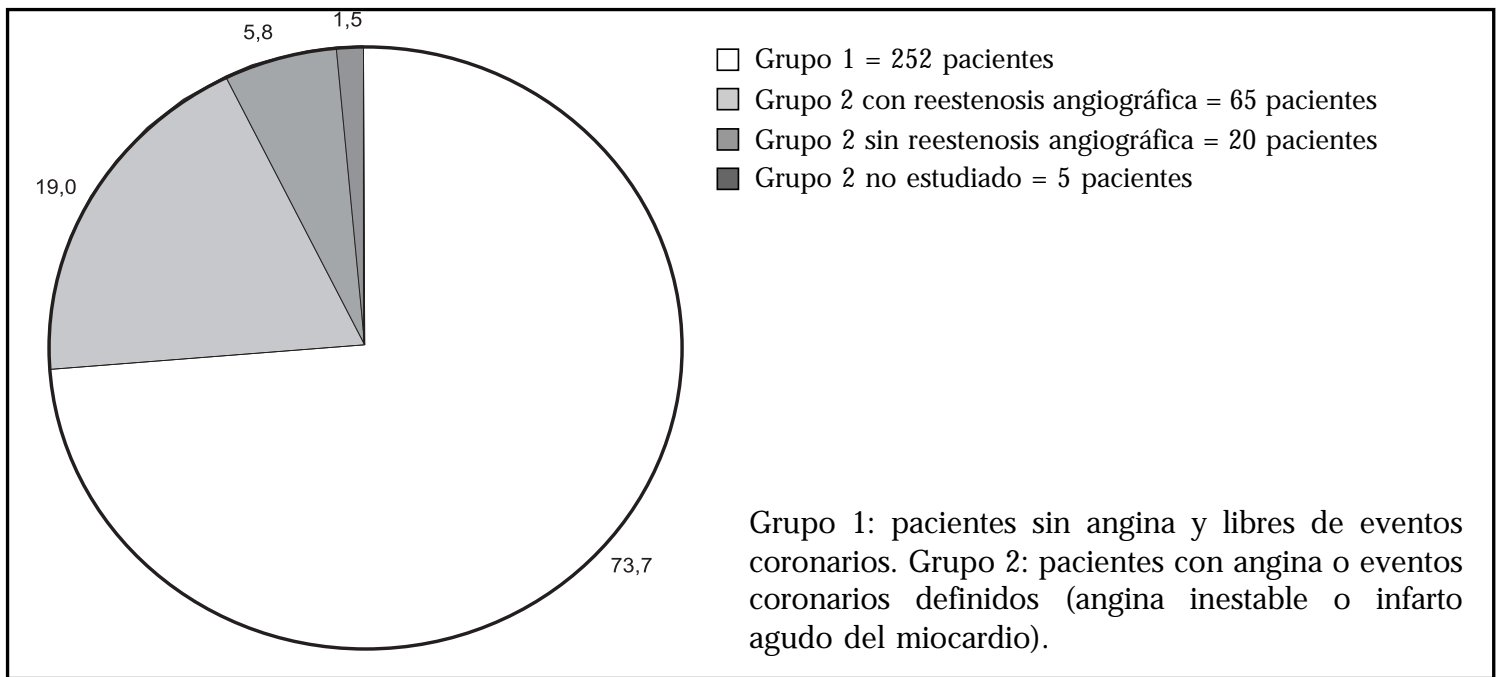

Figura 2. Porcentajes de distribución de los 342 pacientes con angioplastía seguidos a un año.

Tabla 3. Variables clínicas relacionadas con reestenosis de los 342 pacientes seguidos a un año

\begin{tabular}{|llrrrr|}
\hline Variable clínica & O.R & $\mathrm{Z}$ & $\mathrm{p}$ & I.C $95 \%$ & \\
\hline Sexo & 1,27 & 0,68 & 0,494 & 0,$63 ; 2,56$ & $\mathrm{NS}$ \\
Diabetes & 3,46 & 4,04 & $<0,0001$ & 1,$90 ; 6,33$ & $\mathrm{~S}$ \\
Hipertensión arterial & 1,40 & 1,07 & 0,287 & 0,$75 ; 2,62$ & NS \\
Hipercolesterolemia & 1,84 & 1,96 & 0,050 & 1,$0 ; 3,34$ & $\mathrm{~S}$ \\
Obesidad & 2,03 & 2,05 & 0,041 & 1,$03 ; 4,01$ & $\mathrm{~S}$ \\
Tabaco & 1,27 & 0,77 & 0,443 & 0,$69 ; 2,35$ & NS \\
Antecedentes de infarto & 2,51 & 2,68 & 0,007 & 1,$28 ; 4,91$ & $\mathrm{~S}$ \\
\hline
\end{tabular}

O.R: Odds Ratio. I.C 95\%: Intervalo de confianza de 95\%. NS: No Significativo. S: Significativo. 
en bifurcación) en la incidencia de reestenosis (Figura 3). Sólo al analizar la subpoblación de diabéticos, el tipo de lesión adquirió significación estadística, siendo mayor en las complejas $(\mathrm{A}+\mathrm{B} 1$ $=24,2 \%$ vs $\mathrm{B} 2+\mathrm{C}=75,8 \% ; \mathrm{p}=0,0474$ ), $\mathrm{y}$ en las lesiones en bifurcación $(52,4 \%$ de reestenosis en lesiones de bifurcación versus el 31,4\%; p $=0,0399$ ) (Figura 4).

La presencia de stent fue factor protector contra la reestenosis (Figura 5). Esta se presentó en 25,4\% de las lesiones tratadas sin stent (30 de 118 pacientes) y en 15,6\% con stent (35 de 224 pacientes; $\mathrm{p}=0,0141)$. Su protección sólo mostró una tendencia hacia la significancia estadística en la subpoblación de diabéticos con $48,15 \%$ de reestenosis en las lesiones sin stent y $31,25 \%$ con stent ( $p$ $=0,0618$ ), siendo mayor la protección en las lesiones menos complejas ( $16 \%$ en las lesiones A+B1 versus $41,02 \%$ en lesiones $B 2+C$; $p=0,0176$ ). Esta diferencia no se demostró en la subpoblación de no diabéticos donde la incidencia de reestenosis en las lesiones con stent no varió de acuerdo al tipo de

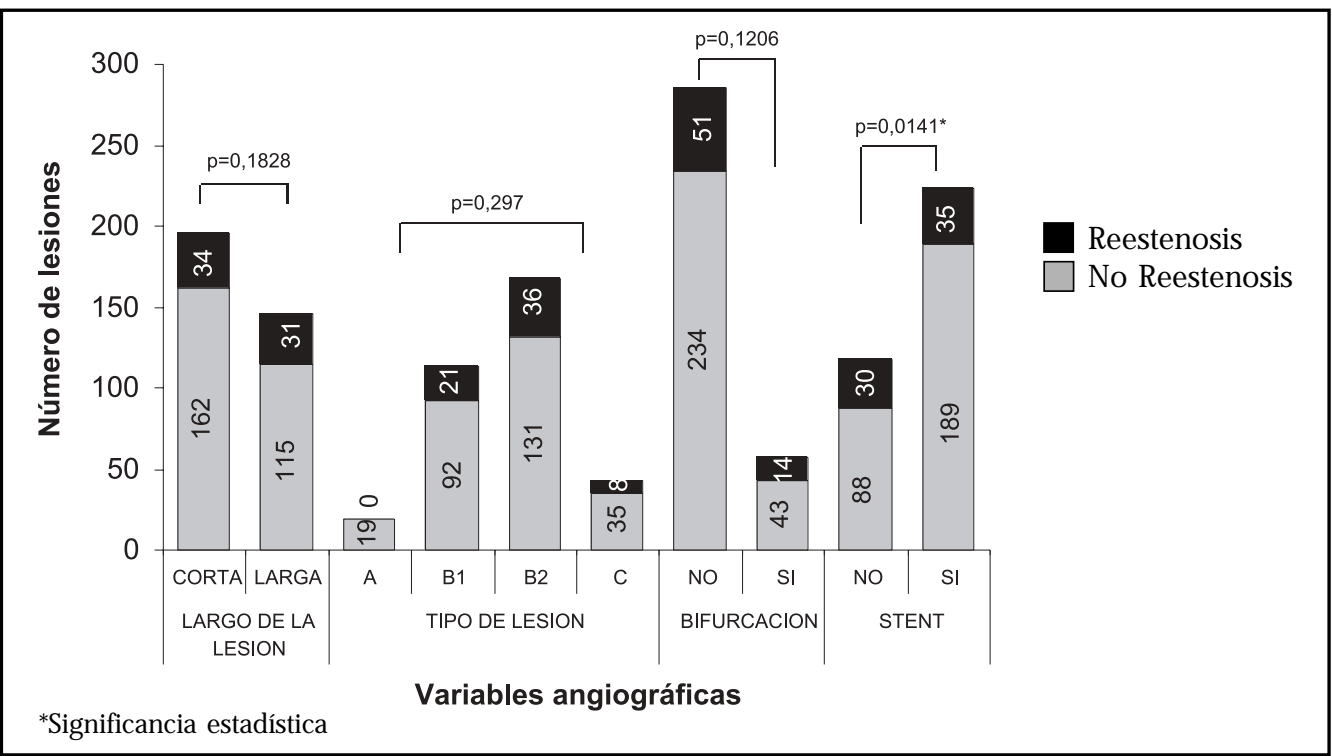

Figura 3. Variables angiográficas y reestenosis

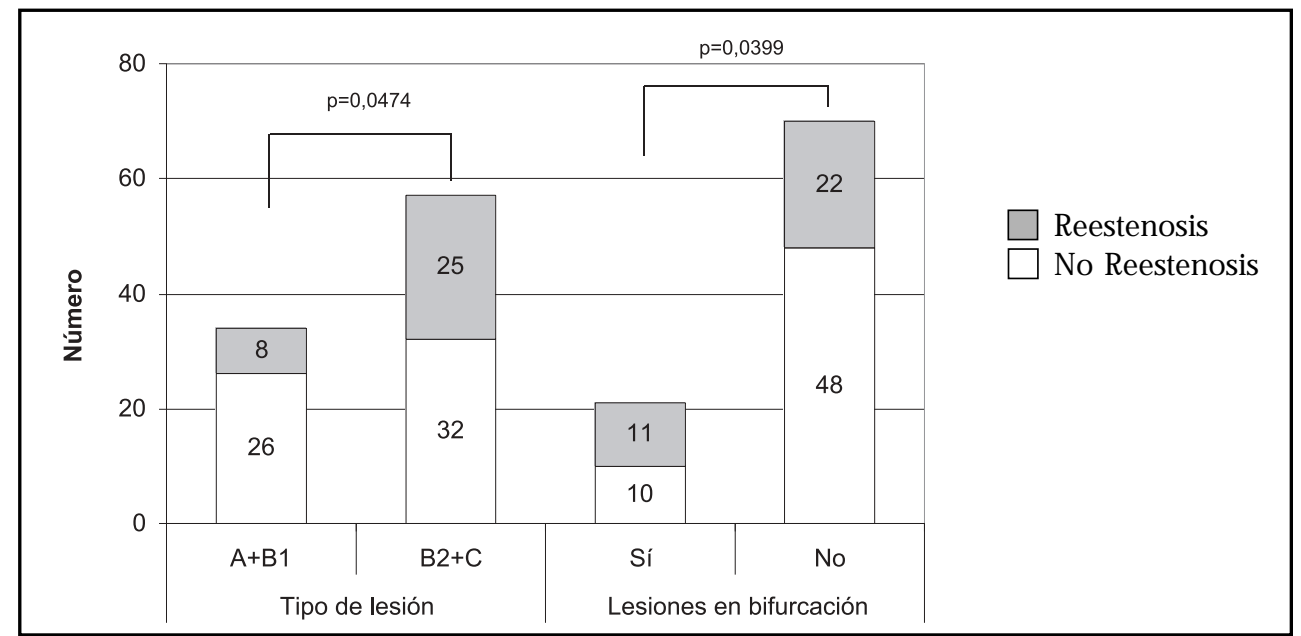

Figura 4. Variables angiográficas relacionadas con reestenosis en diabéticos. 


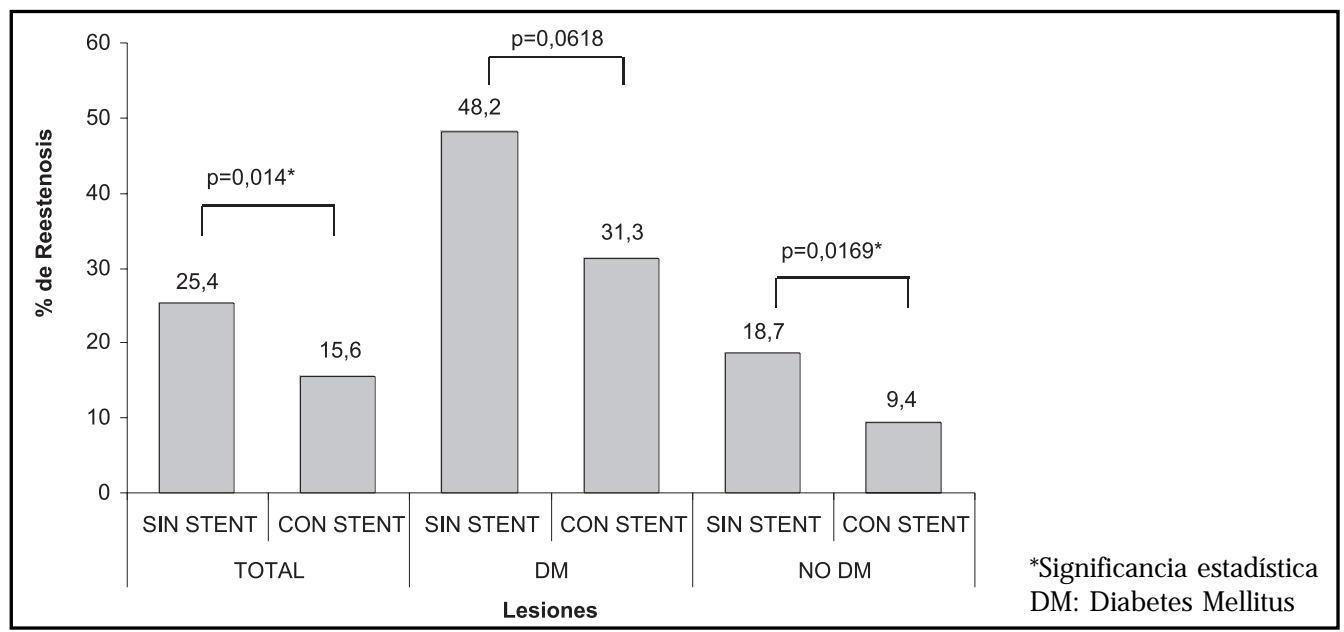

Figura 5. Reestenosis y stent.

lesión (10,17\% en las lesiones $A+B 1$ versus 8,9\% en las $B 2+C ; p=0,3951$ ) (Figura 6).

\section{DiscUSIÓN}

Las características clínicas de nuestra población que llega a ACTP es similar a la de trabajos internacionales y nacionales ${ }^{12-19}$, a excepción de dos variables que son sustancialmente diferentes. Nuestro porcentaje de pacientes dislipidémicos es menor y el antecedente de infartos del miocardio es mayor. El segundo se explica al considerar «antecedente» de infarto todo evento ocurrido hasta la fecha de la coronariografía diagnóstica, lo que suma los infartos agudos, recientes y antiguos en un mismo grupo. Basándonos en datos de otros estudios ${ }^{12,20}$, nuestra cifra pudiera estar sobrevalorada en $10 \%$ a $20 \%$ en relación a infartos agudos.

Para explicar nuestro bajo nivel de pacientes dislipidémicos, tenemos que considerar las carac-

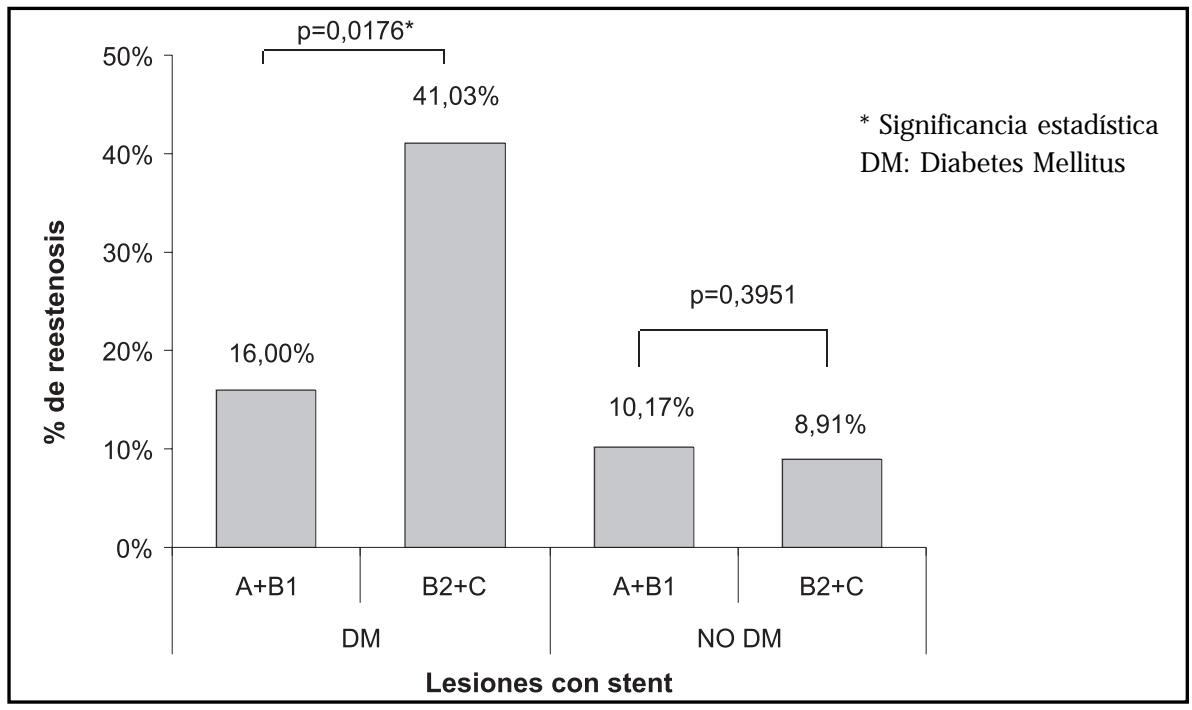

Figura 6. Reestenosis por tipo de lesión, diabetes y stent. 
terísticas generales de la población a la que presta servicio el hospital. Suponemos que el bajo nivel de atención primaria y medicina preventiva hacen que un porcentaje significativo de pacientes presenten un primer evento coronario sin conocimiento ni control de los factores de riesgo coronarios. Además, el estudio basó el diagnóstico de dislipidemia en los antecedentes y no incluyó su búsqueda dirigida durante el período de la coronariografía diagnóstica. Sin embargo, esto no parece ser un problema exclusivo de nuestro diseño de estudio. Las cifras de dislipidemia en la población general conocidas en Chile, publicadas en la primera encuesta nacional de salud de 2003 por el Ministerio de Salud y que incluía la determinación sanguínea de colesterol apuntan a $35,4 \%{ }^{21}$ y en un subgrupo de la población con cardiopatía isquémica, específicamente, la población con infarto agudo del miocardio, es de $30 \% 22$. Otros trabajos nacionales, como el de Dusaillant y cols ${ }^{19}$, también con cuantificación sanguínea de colesterol, reflejan cifras similares a lo publicado internacionalmente (52\%). No tenemos explicación para esta diferencia de resultados en estudios nacionales.

Las características angiográficas de las lesiones descubiertas en nuestra población son similares a lo comunicado en otras series ${ }^{12,14,16,18,20}$. La utilización de stent ha ido en incremento desde su incorporación. En los años de estudio que incluye el trabajo, 65,5\% de ACTP con utilización de stent se encuentra dentro de lo reportado para la época ${ }^{23-24}$.

Nuestra incidencia de reestenosis angiográfica en pacientes con angina o eventos coronarios posangioplastia (Grupo 2) fue de 19\%. Este porcentaje se mantiene en lo reportado en la literatura mundial ${ }^{13,14,21,25}$. Varios estudios han demostrado que la reestenosis angiográfica real se duplica si se estudia también la población asintomática (Grupo 1) y esto deriva en un mayor número de procedimientos de revascularización, sin embargo, la utilidad de tal práctica no está demostrada en ausencia de isquemia13,17,26. Otro dato importante derivado del estudio es que la probabilidad de reestenosis en los pacientes que presenten angina o evento coronario después de una ACTP es muy alto (76,47\%), definiéndola como la primera causa de isquemia sintomática durante el primer año post-ACTP, muy por encima de otras como progresión de la enfermedad aterosclerótica y nuevas lesiones.
El resultado de mayor interés de nuestro estudio es revelar el impacto de la DM en la reestenosis de nuestra población. La DM es la variable más relacionada con la reestenosis, de forma sistemática, también en las series internacionales $^{1-5}$ y sus mecanismos relacionados a un aumento de la hiperplasia neointimal ${ }^{27}$, una respuesta fibrótica acelerada ${ }^{28}$ y una mayor predisposición a la trombosis vascular ${ }^{29}$. Nuestras estadísticas muestran que la DM prácticamente duplica la incidencia de reestenosis con respecto a la población total (36,26\% vs $19 \%$ ) y la triplican si se compara con la muestra de no diabéticos $(12,75 \%)$. La misma relación se mantiene aun con la presencia de stent ( $31,25 \%$ vs $15,63 \%$ vs $9,38 \%$, respectivamente). Variables angiográficas no relacionadas con la reestenosis en nuestra población, como es el tipo de lesión y lesiones en bifurcación y que analizamos posteriormente, adquieren significancia estadística para reestenosis ante la presencia de DM. La reestenosis puede ser tan elevada, que basado en los datos de este estudio, el stent debería utilizarse en toda ACTP de pacientes diabéticos.

Otras variables clínicas también se correlacionaron con la reestenosis, las cuales se repiten en otros trabajos ${ }^{12,17}$. Nuestra diferencia radica en la importancia estadística que demuestran, superior a las variables angiográficas estudiadas y a pesar de la sospecha de una subestimación de la dislipidemia. Queda por determinar si la falta de control de estas variables posprocedimiento, como sospechamos en nuestra población, explicaría su importancia.

En nuestra población, las variables angiográficas analizadas no se relacionan con la incidencia de reestenosis. El tipo de lesión es una clasificación morfológica que ha mostrado su utilidad para predecir éxito del procedimiento y no reestenosis en la mayoría de los estudios ${ }^{10,11,20}$. El largo de la lesión ${ }^{30-32}$ y lesión en bifurcación ${ }^{9,33}$ han sido variables predictoras de reestenosis en algunas series, no así en la nuestra. La literatura actual avala otras variables angiográficas como predictoras de reestenosis y que no fueron analizadas por nosotros, como son el diámetro de referencia, diámetro luminal, longitud, número de stents por lesión, etc ${ }^{16,17}$. Existen evidencias que señalan que a un mejor resultado angiográfico, menor incidencia de reestenosis y que el stent le agrega 
al procedimiento la mayor ganancia angiográfica inicial y previene la retracción elástica y el remodelamiento negativo del vaso tratado ${ }^{34,35}$. Consideramos que nuestro análisis de las variables angiográficas no fue detallado y esto explica la falta de correlación con la reestenosis.

La protección del stent frente a la reestenosis es un hecho confirmado por grandes series ${ }^{13,14,36}$. Nuestro estudio obtiene similares valores a los ya reportados. Frente al paciente diabético, como ya comentamos, su protección disminuye, demostrando que no es una solución definitiva en este subgrupo, pero indiscutiblemente superior a su no utilización.

Limitaciones del estudio. Las variables clínicas fueron recolectadas de forma retrospectiva. Esto pudo ser determinante en nuestra valoración de la dislipidemia, como hemos comentado, pero también en otras variables, como la obesidad, generalmente basado, en nuestro medio, en una estimación visual del examinador. Sólo consideramos isquemia a la isquemia clínica (angina o infarto). Desconocemos la incidencia de isquemia silente y su importancia en nuestra población, demostrado como frecuente e importante en trabajos recientes ${ }^{37,38}$. La evaluación de los parámetros angiográficos no fue detallada. Los diseños

\section{REFERENCIAS}

1. Van Bele E, Abolmaal $K$, Bauters $C$, McFadden EP, LabLANCHe JM, BERTRAND ME. Restenosis, late vessel occlusion and left ventricular function six months after balloon angioplasty in diabetic patients. J Am Coll Cardiol 1999; 34: 476-85.

2. Weintraub WS, Kosinski AS, Brown CL, King SB. Can restenosis after coronary angioplasty be predicted from clinical variables? J Am Coll Cardiol 1993; 21: 6-14.

3. Van Belle E, Bauters C, Hubert E, Bodart JC, Abolmaali K, Meurice T et al. Restenosis rates in diabetic patients: a comparison of coronary stenting and balloon angioplasty in native coronary vessels. Circulation 1997; 96: 1454-60.

4. Eifzi S, Kastrati A, Pache J, Wehinger A, Hadamitzky M, DiRSCHINGER J ET AL. Diabetes mellitus and the clinical and angiographic outcome after coronary stent placement. J Am Coll Cardiol 1998; 32: 1866-73. actuales deben incluir una amplia gama de variables que han demostrado su valor predictor de reestenosis.

En conclusión, las características clínicas y angiográficas de la población con cardiopatía coronaria y ACTP es similar a la de series internacionales a excepción de una menor incidencia de dislipidemia y de la cual sospechamos un bajo pesquisaje en la población general.

La reestenosis en pacientes con angina 0 eventos coronarios posangioplastia en nuestra población asciende a 19\%. Las variables clínicas que influyeron en la incidencia de reestenosis fueron la diabetes mellitus, infarto previo, obesidad y marginalmente la dislipidemia. El factor más importante es la diabetes mellitus.

Las variables angiográficas estudiadas no se asociaron a mayor incidencia de reestenosis. La presencia de DM implicó un mayor riesgo de reestenosis, especialmente en lesiones más complejas y en bifurcación. El uso de stent disminuye la incidencia de reestenosis tanto en DM como en no DM, aunque en los primeros el beneficio aparece significativo sólo en las lesiones menos complejas.

Por último, la reestenosis es la primera causa de angina o infarto en el primer año posangioplastia.

5. Van Belle E, Périé M, Braune D, Chmait A, Meurice T, ABolmaAL $\mathrm{K}$ eT al. Effects of coronary stenting on vessel patency and long-term clinical outcome after percutaneous coronary revascularization in diabetic patients. J Am Coll Cardiol 2002; 40: 410-7.

6. Hamasaki $\mathrm{N}$, Nosaka $\mathrm{H}$, Kimura $\mathrm{T}$, Nakagawa $\mathrm{Y}$, YoKoi $\mathrm{H}$, TAMURA $\mathrm{T}$ eT aL. Influence of lesion length on late angiographic outcome and restenotic process after successful stent implantation (abstr.) J Am Coll Cardiol 1997; 29 Suppl A: 239A.

7. Euss SG, Savage M, Fischman D, Baim DS, León M, Goldberg S et al. Restenosis after placement of Palmaz-Schatz stents in native coronary arteries: initial results of a multicente experience. Circulation 1992; 86: 1836-44.

8. Lablanche J-M, Danchin N, Grower G, Bonnet J-L, Bedossa M, Vahanian A et al. Factors predictive of restenosis after stent implantation managed by ticlopidine and aspirin (abstr). Circulation 1996; 94 Suppl I: I-256. 
9. Weinstein JS, Baim DS, SipperLy ME, McCabe CH, LOREL BH. Salvage of branch vessels during bifurcation lesion angioplasty: acute and longterm follow-up. Cathet Cardiovasc Diagn 1991; 22: 1-6.

10. Ryan TJ, Faxon DP, GunNar RM, Kennedy JW, King SB $3^{\text {RD }}$, Loop FD ET AL. Guidelines for percutaneous transluminal coronary angioplasty. A report of the American College of Cardiology/American Heart Association Task Force on Assessment of Diagnostic and Therapeutic Cardiovascular Procedures (Subcommittee on Percutaneous Transluminal Coronary Angioplasty). Circulation 1988; 78: 486502.

11. Elus SG, Vandormael MG, Cowley MJ, Disciascio G, Deligonul U, Topol EJ et al. Coronary morphologic and clinical determinants of procedural outcome with angioplasty for multivessel coronary disease. Circulation 1990; 82: 1193-202.

12. Mathew V, Grill DE, Scott CG, GarRatt K, Holmes D JR. Baseline clinical and angiographic variables associated with long-term outcome after successful intracoronary stent implantation. Am J Cardiol 1999; 84: 789-94.

13. Serruys PW, de Jaegere P, Kiemeneij F, Macaya C, Rutsch W, Heyndrickx G ET AL, the BENESTENT Study Group. A comparison of balloon-expandable-stent implantation with balloon angioplasty in patients with coronary artery disease. N Engl J Med 1994; 331: 489-95.

14. Serruys PW, van Hout B, Bonnier H, Legrand V, García E, Macaya C et al. Randomized comparison of implantation of heparin-coated stents with balloon angioplasty in selected patients with coronary artery disease (BENESTENT II). Lancet 1998; 352: 673-81.

15. De Jaegere P, Mudra H, Figula H, Almagor $Y$, Doucet S, PenN I et al. IVUS guided optimized stent deployment: immediate and 6 months clinical and angiographic results from the Multicenter Ultrasound Stenting in Coronaries study (MUSIC). Eur Heart J 1999; 19: 1214-23.

16. Tatsuro A, Issam $M$, Bernhard $R$, Ferraro $M$, Kobayashi Y, Blengino S et al. Angiographic and clinical outcome following coronary stenting of small vessels (A comparison with coronary stenting of large vessels). J Am Coll Cardiol 1998; 32: 1610-18.

17. Donald EC, Manish SC, Donald SB, Ho KKL, Popma JJ, Carrozza JP Et aL. Clinical restenosis after coronary stenting: perspectives from multicenter clinical trials. J Am Coll Cardiol 2002; 40: 2082-9.

18. Holmes D JR, Leon M, Moses J, Popma J, Cutlip D, Fitzgerald P ET AL. Analysis of 1-year clinical outcomes in the SIRIUS trial (A randomized trial of a Sirolimus-eluting stent versus a standard stent in patients at high risk for coronary restenosis). Circulation 2004; 109: 634-40.

19. Dussailantt G, Marchant E, Donoso $H$, Aninat M, Opazo M, Pumarino R y cols. Práctica contemporánea de la angioplastia coronaria en Chile: Informe final del Registro Nacional de Angioplastia Coronaria (RENAC) 2001-2002. Rev Méd Chile 2004; 132: 913-22.

20. Zaacks SM, Aluen Je, Calvin Je, Schaer GL, Palvas BW, Parrilo JE et al. Value of The American College of Cardiology/American Heart Association Stenosis Morphology Classification for Coronary Intervention in the late 1990s. Am J Cardiol 1998; 82: 43-9.

21. Primera Encuesta Nacional de Salud. Chile 2003. Ministerio de Salud. Gobierno de Chile. www.epi.minsal.cl/epi/html/invest/ ENS/ InformeFinalENS.pdf

22. GruPo GEMI. Registro del Infarto Agudo del Miocardio en Hospitales Chilenos. 1993-2003.

23. Holmes DR, BeLl MR, Holmes DR $3^{\text {RD }}$, Berger PB, BRESNAHAN JF, HAMMES LN ET AL. Interventional cardiology and intracoronary stents-a changing practice: approved vs nonapproved indications. Cathet Cardiovasc Diag 1997; 40: 133-8.

24. American Heart Association. Heart Disease and Stroke Statistics-2003 Update. Dallas, TX: American Heart Association; 2002.

25. Dan G, AmeEt B, David JC. Can we afford to eliminate restenosis? (can we afford not to?). J Am Coll Cardiol 2004; 43: 513-8.

26. Zellweger MJ, Weinbacher M, Zutter AW, Jeger RV, Muelier-Brand J, Kaiser C et al. Long-Term outcome of patients with silent versus symptomatic ischemia six months After Percutaneous Coronary Intervention and Stenting. J Am Coll Cardiol 2003; 42: 33-40.

27. Kornowski R, Mintz GS, Kent KM, Pichard A, SAtLer L, BUCHER T ET AL. Increased restenosis in diabetes mellitus after coronary interventions is due to exaggerated intimal hyperplasia: a serial intravascular ultrasound study. Circulation 1997; 95: 1366-9.

28. Moreno PR, Fallon JT, Murcia AM, Leon MN, Simosa H, Fuster V et al. Tissue characteristics of 
restenosis after percutaneous transluminal coronary angioplasty in diabetic patients. J Am Coll Cardiol 1999; 34: 1045-9.

29. Davi G, Catalano I, Averna M, Notarbartolo A, Strano A, Ciabattoni G et al. Thromboxane biosynthesis and platelet function in type II diabetes mellitus. N Engl J Med 1990; 322: 176974.

30. Hirshfeld JW, Schwartz JS, Jugo R, MacDonald RG, Goldberg S, Savage MP ET AL, for the M-HEART Investigators. Restenosis after coronary angioplasty. J Am Coll Cardiol 1991; 18: 647-56.

31. Bauters C, Hubert E, Prat A, Bougrimi K, Van Belle E, McFadden EP ET aL. Predictors of restenosis after coronary stent implantation. J Am Coll Cardiol 1998; 31: 1291-8.

32. Kobayashi $Y$, De Gregorio J, Kobayashi N, Akiyama T, REIMERS B, Finci L ET AL. Stented segment length as an independent predictor of restenosis. J Am Coll Cardiol 1999; 34: 651-9.

33. Al Suwaidi J, Berger PB, Ruhal CS, Garratt K, Bell $\mathrm{M}$, TING $\mathrm{H}$ ET AL. Inmediate and long term outcome of intracoronary stent implantation for true bifurcation lesions. J Am Coll Cardiol 2000; 35: 929-36.

34. Kuntz RE, Safian RD, Carrozza JP, Fishman RF, Mansour M, BAIM DS. The importante of acute luminar diameter in determining restenosis after coronary atherectomy or stenting. Circulation 1992; 86: 1827-35.

35. Kuntz RE, Gibson CM, Nobuyoshi M, Baim DS. Generalized model of restenosis after conventional balloon angioplasty, stenting and directional atherectomy. J Am Coll Cardiol 1993; 21: 15-25.

36. Betriu A, Masotti M, Serra A, Alonso J, FernándezAvilés F, Gimeno F et al. Randomized comparison of coronary stent implantation and balloon angioplasty in the Treatment of De Novo Coronary Artery Lesions (START): a four-year follow-up. J Am Coll Cardiol 1999; 34: 1498-506.

37. Ruygrok PN, Webster MWI, de Valk V, van Es G-A, Ormiston J, Morel M-A et al. Clinical and angiographic factors associated with asymptomatic restenosis after percutaneous coronary intervention. Circulation 2001; 104: 2289-94.

38. Steven RB, Kenneth NG. Silent Ischemia: Unsafe at Any Time. J Am Coll Cardiol 2003; 42: 41-4. 